\title{
Health care professionals' attitudes regarding patient safety: cross-sectional survey
}

\author{
Indre Brasaite ${ }^{1,3^{*}}$, Marja Kaunonen ${ }^{1,2}$, Arvydas Martinkenas ${ }^{3}$ and Tarja Suominen ${ }^{1}$
}

\begin{abstract}
Background: Patient safety is being seen as an increasingly important topic in the healthcare fields, and the rise in numbers of patient safety incidents poses a challenge for hospital management. In order to deal with the situation, it is important to know more about health care professionals' attitudes regarding patient safety. This study looks to describe health care professionals' attitudes regarding patient safety, and whether differences exist based on the background factors of study participants.

Methods: A quantitative study using a questionnaire was conducted in three multi-disciplinary hospitals in Western Lithuania. Data was collected in 2014 from physicians, nurses and nurse assistants.

Results: The results showed positive safety attitudes, and these were especially related to the respondents' levels of job satisfaction. A respondent's older age was associated with how they evaluated their teamwork climate, safety climate, job satisfaction, and perception of management. Profession, working unit, length of work experience, information received about patient safety during education, further education, and working shifts were all associated with several safety attitude areas.

Conclusions: The safety attitudes of respondents were generally found to be positive. Attitudes related to patient safety issues were positive among health care professionals and opens the door for the open discussion of patient safety and adverse events. However, in future we also need to investigate the knowledge and skills professionals have in relation to patient safety, in order to gain a deeper understanding of the present situation.
\end{abstract}

Keywords: Attitude, Health care professionals, Nurses, Patient safety, Physicians, Nurse assistants

\section{Background}

Attitudes regarding safety-related issues are an important part of what is often called a hospital's safety culture $[1,2]$. An organization's safety culture consists of components concerning healthcare provider attitudes about organizational factors such as safety climate and morale, work environment factors such as staffing levels and managerial support, team factors such as teamwork and supervision, and staff factors such as overconfidence and being overly self-assured [3]. Some authors [4-6] have noticed that a safety culture is a part of the wider organisational culture, and may be defined as the attitudes, beliefs, perceptions, competencies and values

\footnotetext{
*Correspondence: Brasaite.Indre.X@student.uta.fi

${ }^{3}$ Faculty of Health Sciences, Klaipeda University, Klaipeda, Lithuania

Full list of author information is available at the end of the article
}

that determine an organisation's health and safety management, and are held in common by employees in relation to safety. An understanding of nurses' perceptions and expectations regarding adverse events is therefore essential for the implementation of appropriate strategies to manage nursing care. In this sense, the beliefs, values and organizational culture of registered nurses (RNs) are important aspects to be considered [7].

Ethical issues are integral to the topic of patient safety because it is known that millions of patients worldwide suffer injury or death every year as a result of unsafe medical practices and care, and patients are mostly harmed due to preventable causes that they receive during health care in hospital settings [8]. Health care professionals may know that their role is important in the delivery of safe care and that they should have positive safety attitudes. However, the results of a safety culture 
study [9] showed that both RNs and nurse managers were critical of the state of patient safety in acute care hospitals, with RNs being the more critical group. That said, generally positive attitudes to patient safety have been reported among health care professionals [10], and the safety climate within healthcare has been evaluated more positively by physicians than nurses [11].

Previous literature has shown some differences in attitudes regarding patient safety, based on profession, age, gender and working area. In one study, the connection of safety attitudes to profession was measured by researchers [12]. The results showed that only $39 \%$ of physicians had a positive attitude towards safety climate, and less than half of the physicians and nurses surveyed were satisfied with their jobs (47 and $45 \%$, respectively). Physicians, nurses, and medical assistants had relatively similar but low perceptions of their working conditions when compared to managers $(29,36$, and $35 \%$, respectively). Researchers have explored professional differences in patient safety attitudes among operating room (OR) care givers in nine medical centres [13]. Of the six patient safety domains covered in the study, stress recognition and working conditions showed significant differences by univariate analysis of profession. Regression analysis revealed that differences for job satisfaction and working conditions were seen among the professions studied. In intensive care units, surgeons have expressed more favourable perceptions of working conditions than nurses [14], and surgeons have also been seen to have a more favourable perception of management than OR nurses [13].

In a study conducted in the field of obstetrics, the highest positive safety attitudes score $(48.3 \%)$ was reported by the $30-35$ years age group of health care professionals [15]. Associations between gender differences and patient safety attitudes are also to be found in the literature. Gender differences in patient safety attitudes were explored among OR care givers, and of the six patient safety scales, four showed significant differences in univariate analysis (teamwork climate, job satisfaction, perceptions of management, and working conditions). Women were found to have less favourable perceptions of teamwork [69, versus (vs) 76 for men], job satisfaction (74, vs 80 for men), management (60, vs 69 for men), and working conditions (57, vs 72 for men) [13].

Work area and discipline have also been reported to be associated with attitudes [4]. One of the key findings was that emergency department (ED) personnel, particularly ED nurses, perceived substantially lower levels of safety climate than workers in other areas. This suggests that the higher levels of risk and complexity, and the faster pace associated with work performed in emergency departments continue to require relatively more attention to be paid to safety issues than other areas.
To maintain a safe patient environment and safe practices, it is very important to promote the measurement and improvement of safety attitudes among health care professionals [16]. The research presented in this paper looks to describe health care professionals' attitudes regarding patient safety, and whether differences exist based on the background factors of the study participants.

\section{Methods}

\section{Data collection}

The study was carried out in three hospitals in one region of Lithuania, and involved all of the health care professionals (physicians, head nurses, nurses and nurse assistants) who worked with adult patients. The hospitals involved are of similar size and provide multi-profile care for Western Lithuanian residents. The criteria for inclusion in the research were that participants were health care professionals, working in health care organizations (hospitals) with adult patients, and would participate voluntarily in the study.

Data was collected using a questionnaire consisting of background questions based on existing literature, and an instrument measuring patient safety attitudes. Twenty background questions investigated the basic demographic characteristics of participants (e.g. work position, place of work, age, gender, education, years at work, usual shift, working hours per week), as well as information concerning the type and hours of training they had received regarding patient safety. Finally, participants were asked how many adverse events they had reported during the previous year. They were also asked what kind of patient safety related events they had faced, and whether they had reported them.

The data for measuring safety attitudes was collected using the University of Texas safety attitudes questionnaire (SAQ) [3] (short form version) that consists of six scales: teamwork climate, safety climate, perceptions of management, job satisfaction, working conditions, and stress recognition. Additional to the SAQ, five further statements examining safety attitudes were included, such as the health care professionals' perceptions of whether safety issues would be acted upon if they expressed them to management, and whether they experienced good collaboration with other nurses, staff physicians and pharmacists in their clinical area. A final statement examined if communication breakdowns that lead to delays in the delivery of care were common. The SAQ (short form version) used in this study comprised of 36 items, each answered using a six-point Likert scale: $1=$ disagree strongly, $2=$ disagree slightly, $3=$ neutral, $4=$ agree slightly, $5=$ agree strongly, and $6=$ not applicable. Negatively worded items were reverse scored so that their valence matched the positively worded items. 
The SAQ (short form version) was used because of its usability, the good psychometric properties it has shown in previous studies, and its broad potential for implementation [3, 17]. The instrument was originally developed in the United States of America and was translated from English into Lithuanian using the back-translation technique [18]. Permission to use the instrument in this study was obtained from the copyright holder of the instrument by the one of the authors. The questionnaire was piloted in one regional hospital with health care professionals to evaluate the validity of the instrument, and also its use in the Lithuanian context. The pilot data collection took place in February 2014, and included the hospital staff $(\mathrm{n}=90)$. The pilot study hospital was not included in the main study. The SAQ showed good psychometric properties. The scales reliability was assessed with a total Cronbach's alpha of .78, corrected by interitem correlation from .05 to .69. The Cronbach's alpha values were good for all scales for the main (and pilot) study: for teamwork climate $.62(.66)$, safety climate .74 (.78), job satisfaction $.87(.86)$, stress recognition .79 (.88), perceptions of management $.90(.92)$, and Working Conditions .74 (.78).

Ethical approval for the study was obtained from the Ethics Committee of Klaipeda University, Faculty of Health Sciences (Nr. $46 \mathrm{~Sv}-\mathrm{SL}-1$ ), and permission to collect data was also obtained from the hospitals participating in the pilot and main phases of the study. The ethical considerations related to the data collection were focused on the ethical principles for research, namely confidentiality (related to questionnaires), privacy, and the voluntary nature of participation in the study [19].

The main study data was collected in May 2014 in three regional hospitals. In each hospital, questionnaires with return envelopes were delivered to established contact persons. The contact persons circulated the questionnaires to all staff $(n=1687)$. After 2 weeks, the researcher collected the returned questionnaires in sealed envelopes from each unit. Because of a low response rate [46\% $(\mathrm{n}=774)$, reminder letters were left for the contact persons who were asked to circulate them. An additional 2 weeks were given to respond, and the final response rate was $64 \%(n=1082)$.

\section{Statistical analyses}

Descriptive statistics were used to describe the characteristics of respondents (physicians, head nurses, nurses and nurse assistants), the SAQ items, and the scale-level results of the three hospitals. Differences in sample characteristics between hospitals and professional groups were tested using one-way analysis of variance (ANOVA) and the Tukey HSD (honest significant difference) multiple comparison test, or the Tamhane multiple comparison test (when the assumption of equal variances was not correct). Non-normally distributed characteristics were analysed using the Kruskal-Wallis test. Data was presented using mean [standard deviation $(\mathrm{SD})$ ] or median [interquartile range (IQR)] expression. Any negatively worded items of the SAQ were reversed before analysis. The internal consistency of the SAQ and its scales of safety climate, job satisfaction, perception of management, and working conditions (for SAQ) was measured by calculating the Cronbach's alpha for each area. Associations between variables were calculated by means of Spearman correlations.

For further analysis, the units in which the respondents worked were re-grouped as internal medicine (e.g. internal diseases, neurology, cardiology, heart arrhythmia, haemodialysis, nephrology etc.), surgical (e.g. surgery, traumatology), psychiatric (e.g. mental health, treatment of addiction), acute (e.g. resuscitation, anaesthesiology, emergency, operating room, intensive care), and others (e.g. rehabilitation, laboratory, polyclinics etc.). Head nurses and nurses were also combined into one group (756 nurses including 54 head nurses). All of the data was analyzed using SPSS (Statistical package for social sciences) (version 22.0; SPSS Inc., Chicago, Illinois, USA). A $p$ value of $<.05$ was considered to be statistically significant.

\section{Results}

\section{Participants}

The questionnaire was answered by 1082 (64\%) of the health care professionals surveyed. Participants were nurses ( $\mathrm{n}=756,70 \%)$, nurse assistants $(\mathrm{n}=180,16.6 \%)$ and physicians $(\mathrm{n}=146,13.5 \%)$. Most participants were female 989 (91.4\%) and their mother tongue was Lithuanian 1018 (94.1\%). The most common education institutions of the study participants were medical school 493 (45.6\%), college 130 (12.0\%), and a university bachelor programme 118 (10.9\%). Respondents stemmed from three regional hospitals: 301 (27.8 \%) from hospital 1, 411 (38.0 \%) from hospital 2, and 370 (34.2 \%) from hospital 3. The mean age of the participants was 46.7 years $(\mathrm{SD}=10.9)$. Most had a permanent position at their hospital ( $\mathrm{n}=1047,96.8 \%$ ), the mean work experience was more than 20 years $($ mean $=23.9)$, and they worked an average of $39.9 \mathrm{~h}$ per week in their unit. Some health care professionals $(\mathrm{n}=140,12.9 \%)$ had an extra job and worked an average of $18.6 \mathrm{~h}$ per week in this setting. Most of the health care professionals ( $n=659,60.9 \%$ ) worked variable shifts, and in units with averages of 30.7 beds and 24.9 staff members. Usually, one health care professional had 18 patients per working shift. Almost two thirds of the participants $(n=673,62.2 \%$ ) had received no information about patient safety during their initial 
professional education, but about half $(\mathrm{n}=589,54.4 \%)$ had received some in their further education (Table 1). $80 \%$ of respondents $(n=866)$ had not reported any patient safety incidents during the last year.

\section{Safety attitudes}

The results of this study show positive safety attitudes overall, in regard to job satisfaction (mean $=4.14$ ), teamwork and safety climate (mean $=4.10$ in each domain), and working conditions (mean $=4.09$ ) (Table 2). Only in the area of perceptions of management there were differences $(p<.001)$ to be seen between the three hospitals participating in the study.

The most positive safety attitudes represented in the SAQ scales tended to correlate with the most background factors, namely safety climate, job satisfaction, perceptions of management and working conditions. Older health care professionals were associated with how they evaluated teamwork climate (.061), safety climate (.078), their job satisfaction (.150) and their perceptions of management (.140). The length of work experience in general was associated with how participants evaluated their safety climate (.082), job satisfaction (.155) and their perceptions of management (.193). Respondents who had received information about patient safety during their education were associated with how they reported their teamwork climate $(-.090)$, safety climate $(-.093)$, job satisfaction $(-.076)$, perceptions of management $(-.093)$ and working conditions $(-.072)$. Those who had received information about patient safety in continuing education reported the same associations, with the exception of teamwork climate. Whether the health care professional worked day shifts or variable shifts was associated with her/his safety attitudes in all of the investigated safety areas: teamwork climate $(-.108)$, safety climate $(-.089)$, job satisfaction $(-.137)$, stress recognition $(-.088)$, perceptions of management (-.188) and working conditions (-.154) (Table 3).

Physicians had significantly higher safety attitudes related to teamwork climate $(\mathrm{p}=.014)$ and Stress Recognition $(\mathrm{p}<.001)$ than nurses and nurse assistants in the group of health care professionals who did not report a safety incident during the last year, but the attitudes towards the Perceptions of Management $(\mathrm{p}<.001)$ in the same group were significantly higher for physicians and nurse assistants than nurses. In the health care professional group who had reported a safety incident during last year, physicians had significantly higher safety attitudes related to their teamwork climate than nurses and nurse assistants $(\mathrm{p}=.011)$. Those who didn't report any safety incidents during the last year had more positive attitudes towards Stress Recognition than those who had reported such incidents.
Table 1 Work related background factors $(n=1082)$

\begin{tabular}{|c|c|c|}
\hline & Mean (SD) & Median (IQR) \\
\hline Years of experience in primary speciality & $21.61(12.04)$ & $22.00(17)$ \\
\hline Years of work experience in general & $23.88(11.52)$ & $25.00(15)$ \\
\hline Years worked in this unit & $14.32(11.80)$ & $12.00(15)$ \\
\hline Working hours per week in this unit & $39.86(8.23)$ & $38.00(2)$ \\
\hline Hours per week in extra job & $18.61(14.63)$ & $16.50(16)$ \\
\hline Number of beds per unit & $30.72(17.27)$ & $30.00(20)$ \\
\hline Total number of staff working in unit & $24.09(10.33)$ & $23.00(10)$ \\
\hline $\begin{array}{l}\text { Number of patients health care profession- } \\
\text { als usually have per working shift }\end{array}$ & $18.00(12.03)$ & $15.00(12)$ \\
\hline \multicolumn{3}{|l|}{ Health care professionals working in unit } \\
\hline \multicolumn{3}{|l|}{ Day shift } \\
\hline Physicians & $4.28(3.15)$ & $3.00(4)$ \\
\hline Nurses & $4.72(4.82)$ & $3.00(3)$ \\
\hline Nurse assistants & $2.48(2.14)$ & $2.00(2)$ \\
\hline \multicolumn{3}{|l|}{ Evening shift } \\
\hline Physicians & $1.69(1.47)$ & $1.00(1)$ \\
\hline Nurses & $2.34(1.81)$ & $2.00(1)$ \\
\hline Nurse assistants & $1.62(1.08)$ & $1.00(1)$ \\
\hline \multicolumn{3}{|l|}{ Night shift } \\
\hline Physicians & $1.19(.82)$ & $1.00(0)$ \\
\hline Nurses & $1.90(1.15)$ & $2.00(1)$ \\
\hline Nurse assistants & $1.28(.67)$ & $1.00(0)$ \\
\hline Usual shift & $N(\%)$ & \\
\hline Day & $398(36.8)$ & \\
\hline Evening & $2(.2)$ & \\
\hline Night & $7(.7)$ & \\
\hline Variable shifts & $659(60.9)$ & \\
\hline Other & $14(1.3)$ & \\
\hline \multicolumn{3}{|l|}{ Extra job } \\
\hline Yes & $140(12.9)$ & \\
\hline No & $939(86.8)$ & \\
\hline \multicolumn{3}{|c|}{ Information about patient safety during initial education } \\
\hline Yes & $408(37.7)$ & \\
\hline No & $673(62.2)$ & \\
\hline If yes, hours [mean (SD), median (IQR)] & $37.14(58.49)$ & $20.00(30)$ \\
\hline \multicolumn{3}{|c|}{ Information about patient safety in continuing education } \\
\hline Yes & $589(54.4)$ & \\
\hline No & $492(45.5)$ & \\
\hline If yes, hours [mean (SD), median (IQR)] & $24.22(32.02)$ & $14.00(30)$ \\
\hline
\end{tabular}

When comparing safety attitudes between health care professionals by working unit, some significance differences were noted. Health care professionals working in psychiatric units had significantly lower safety attitudes relating to job satisfaction $(\mathrm{p}=.004)$, perceptions of management $(\mathrm{p}<.001)$ and working conditions $(\mathrm{p}<.001)$ than those working in internal medicine, surgical, acute or other units. 
Table 2 Patient safety attitudes

\begin{tabular}{|c|c|c|c|c|}
\hline $\begin{array}{l}\text { SAQ Short form } \\
\text { scales/hospitals }\end{array}$ & Mean (SD) & Median (IQR) & Chi square & $p$ value \\
\hline \multicolumn{5}{|l|}{ Teamwork climate } \\
\hline Hospital 1 & $4.07(.64)^{*}$ & $4.07(.64)^{*}$ & & \\
\hline Hospital 2 & $4.08(.72)$ & $4.08(.72)$ & & \\
\hline Hospital 3 & $4.16(.67)^{*}$ & $4.16(.67)^{*}$ & & \\
\hline Total & $4.10(.68)$ & $4.10(.68)$ & 3.84 & .147 \\
\hline \multicolumn{5}{|l|}{ Safety climate } \\
\hline Hospital 1 & $4.07(.67)$ & $4.07(.67)^{*}$ & & \\
\hline Hospital 2 & $4.05(.72)^{\ddagger}$ & $4.05(.72)^{\ddagger}$ & & \\
\hline Hospital 3 & $4.17(.67)^{\ddagger}$ & $4.17(.67)^{*}$ & & \\
\hline Total & $4.10(.69)$ & $4.10(.69)$ & 7.86 & .020 \\
\hline \multicolumn{5}{|l|}{ Job satisfaction } \\
\hline Hospital 1 & $4.21(.84)^{*}$ & $4.21(.84)^{*}$ & & \\
\hline Hospital 2 & $4.05(.90)^{*}$ & $4.05(.90)^{*}, \neq$ & & \\
\hline Hospital 3 & $4.19(.84)$ & $4.19(.84)^{\ddagger}$ & & \\
\hline Total & $4.14(.87)$ & $4.14(.87)$ & 6.35 & .042 \\
\hline \multicolumn{5}{|l|}{ Stress recognition } \\
\hline Hospital 1 & $3.86(.88)$ & $3.86(.88)$ & & \\
\hline Hospital 2 & $3.79(.94)$ & $3.79(.94)$ & & \\
\hline Hospital 3 & $3.75(1.01)$ & $3.75(1.01)$ & & \\
\hline Total & $3.80(.95)$ & $3.80(.95)$ & 1.12 & .572 \\
\hline \multicolumn{5}{|c|}{ Perceptions of management } \\
\hline Hospital 1 & $3.75(.83)^{*}$ & $3.75(.83)^{*}$ & & \\
\hline Hospital 2 & $3.58(.89)^{*}$ & $3.58(.89)^{*}, \neq$ & & \\
\hline Hospital 3 & $3.83(.88)^{\ddagger}$ & $3.83(.88)^{\ddagger}$ & & \\
\hline Total & $3.71(.88)$ & $3.71(.88)$ & 20.76 & $<.001$ \\
\hline \multicolumn{5}{|l|}{ Working conditions } \\
\hline Hospital 1 & $4.05(.97)$ & $4.05(.97)$ & & \\
\hline Hospital 2 & $4.04(.98)$ & $4.04(.98)^{\ddagger}$ & & \\
\hline Hospital 3 & $4.18(.96)$ & $4.18(.96)^{\ddagger}$ & & \\
\hline Total & $4.09(.97)$ & $4.09(.97)$ & 5.54 & .063 \\
\hline
\end{tabular}

For Means: ANOVA + Tukey HSD multiple comparison test

For Ranks: Kruskal-Wallis Test + Mann-Whitney U comparison test

${ }^{*} \mathrm{p}<.05$-differences between Hospital 1 and Hospital 2 or Hospital 3

$\neq \mathrm{p}<.05$-differences between Hospital 2 and Hospital 3

Nearly two thirds of health care professionals ( $\mathrm{n}=638$, $59 \%)$ felt that their suggestions about safety would be acted upon if they expressed them to management, whilst $20.1 \%(\mathrm{n}=218)$ were neutral and $18.1 \%(\mathrm{n}=195)$ disagreed. Most of the respondents experienced good collaboration with nurses $(\mathrm{n}=914,84.5 \%)$, with staff physicians ( $\mathrm{n}=859,79.4 \%$ ), but less with pharmacists $(\mathrm{n}=248,22.9 \%)$ in their clinical area. Only $18.7 \%$ $(n=203)$ of health care professionals felt that communication breakdowns that lead to delays in the delivery of care were common.

\section{Discussion}

Our goal was to assess the general situation regarding the safety attitudes of health care professionals, because no national-level data had been reported in either Lithuania or any of the other Baltic Countries. Overall, the safety attitudes of health care professionals were positive and in-line with previous studies (e.g. [10, 20-22]). However, whilst the results of this study were partly in-line with earlier results, there were also contradictory elements. As such, further study is needed to establish links between these areas, and the attitudes and background factors of individual respondents, and this may prove important in developing our clinical practices. Age seemed to be associated with many safety attitudes scales, and it has previously been reported [15] that the highest positive safety score when comparing younger and older age groups was to be found to be in the 30-35 year age group. In our study however, safety attitudes were found to be higher in older age groups. This may be explained by the linked years of work experience $($ mean $=23.9)$ which indicates that health care professionals who know their job very well, may also hold enhanced safety attitudes.

Gender was only associated with stress recognition, although a previous study [13] has shown gender to be associated with several safety attitudes such as teamwork climate, job satisfaction, perceptions of management, and working conditions. In our study, only about $10 \%$ of the respondents were male, which may have had an effect on the results.

It was interesting to find that physicians had higher safety attitudes towards teamwork climate than nurses and nurse assistants. this may indicate that physicians tend to value teamwork more when adverse events happen, and perhaps consider the issue to be faced as more of a common responsibility than other health care professional groups. Our result is similar to previous positive physician safety attitudes reported by other researchers, for example where physicians had more positive attitudes in perceptions of their working conditions [14], and in their perceptions of management [13] than nurses.

In comparing safety attitudes between health care professionals by work area, it was found that respondents in psychiatric units had significantly lower safety attitudes than those working in internal medicine, surgi$\mathrm{cal}$, acute and other units. This might be linked to their working environment, as health care professionals may be more stressed when working with patients with mental illnesses, and sometimes be subjected to physical or psychological violence from their patients. Another explanation may be that health care professionals think 
Table 3 Correlations between respondents' background factors and their patient safety attitudes

\begin{tabular}{|c|c|c|c|c|c|c|}
\hline Demographic characteristics & $\begin{array}{l}\text { Teamwork } \\
\text { climate }\end{array}$ & $\begin{array}{l}\text { Safety } \\
\text { climate }\end{array}$ & Job satisfaction & $\begin{array}{l}\text { Stress recogni- } \\
\text { tion }\end{array}$ & $\begin{array}{l}\text { Perceptions of man- } \\
\text { agement }\end{array}$ & $\begin{array}{l}\text { Working } \\
\text { conditions }\end{array}$ \\
\hline Age & $.061^{*}$ & $.078^{*}$ & $.150^{*}$ & -.013 & $.140^{*}$ & .053 \\
\hline Gender & -.041 & .013 & -.017 & $-.083^{*}$ & -.029 & .039 \\
\hline Education & $.101^{* *}$ & .024 & .003 & .059 & $.090^{* *}$ & .044 \\
\hline Years of experience in primary speciality & .007 & .041 & $.086^{* *}$ & -.003 & .036 & .046 \\
\hline Years of work experience in general & .053 & $.082^{* *}$ & $.155^{* *}$ & -.033 & $.093^{* *}$ & .053 \\
\hline $\begin{array}{l}\text { Information about patient safety during initial } \\
\text { education }\end{array}$ & $-.090^{* *}$ & $-.093^{* *}$ & $-.076^{*}$ & -.007 & $-.093^{* *}$ & $-.072^{*}$ \\
\hline $\begin{array}{l}\text { Information about patient safety in continuing } \\
\text { education }\end{array}$ & -.058 & $-.111^{* *}$ & $-.100^{* *}$ & .018 & $-.099^{* *}$ & $-.063^{*}$ \\
\hline $\begin{array}{l}\text { Received hours regarding information about } \\
\text { patient safety in continuing education }\end{array}$ & -.052 & .005 & -.017 & -.039 & .015 & $-.146^{*}$ \\
\hline Usual shift & $-.108^{* *}$ & $-.089^{* *}$ & $-.137^{* *}$ & $-.088^{* *}$ & $-.188^{* *}$ & $-.154^{* *}$ \\
\hline Working hours per week in this unit & -.040 & -.026 & -.054 & $.067^{*}$ & -.059 & -.049 \\
\hline Extra job & .027 & $.081^{* *}$ & .047 & -.046 & .014 & .025 \\
\hline Number of beds per unit & -.038 & $-.074^{*}$ & -.066 & .056 & -.039 & .003 \\
\hline Total number of staff working in unit & -.040 & -.006 & -.009 & $.079^{*}$ & $-.092^{* *}$ & -.057 \\
\hline $\begin{array}{l}\text { Number of physicians working in unit on day } \\
\text { shifts }\end{array}$ & -.024 & .042 & .046 & .039 & .056 & $.110^{* *}$ \\
\hline Number of nurses working in unit on day shifts & .036 & .034 & $.091^{* *}$ & .005 & .046 & $.081^{*}$ \\
\hline $\begin{array}{l}\text { Number of patients health care professionals } \\
\text { usually have per working shift }\end{array}$ & -.020 & -.033 & $-.095^{* *}$ & -.012 & -.053 & $-.078^{*}$ \\
\hline
\end{tabular}

Spearman correlations, ${ }^{*} \mathrm{p}<.05,{ }^{* *} \mathrm{p}<.01$

of safety issues differently depending upon the type of treatment involved (e.g. operations, infections, or patient falls), and some of these issues may not be seen to be so relevant in psychiatric units.

Other researchers [4] have also highlighted differences in attitudes between work areas, with the main finding that ED personnel perceived substantially lower levels of safety climate than workers in other clinical areas.

Health care professionals who received no information about patient safety during their initial professional education had more negative attitudes to teamwork climate, safety climate, job satisfaction, perceptions of management and working conditions than those who had. Also, health care professionals who received no information about patient safety during their further/continued education had lower ranked attitudes to safety climate, job satisfaction, perceptions of management and working conditions than whose who had. Drawing from this, we may consider that education about patient safety impacts upon the safety attitudes of health care professionals.

\section{Methodological considerations}

There are several strengths in this study. Especially, the response rate was good (64\%) and we were able to reach both nurses and physicians in the same study. A limitation may be noted in that the data was purposefully collected from one region. However, we consider these results to present a fairly representative view of patient safety attitudes that may be predicted in similar size multi-profile hospitals across Lithuania, as the country is divided into 10 similar size regions where health care services are organized using the same structure, and serve similar sized populations. Thus the results may readily transpose to a wider setting within Lithuania, but they may not be representative of other national settings.

\section{Conclusions}

Attitudes related to patient safety issues are positive among health care professionals in Lithuania, which helps to open the door for the open discussion of patient safety and adverse events. However, in future we also need to investigate the knowledge and skills professionals have in relation to patient safety, in order to gain a deeper understanding of the present situation.

\section{Abbreviations}

RNs: registered nurses; OR: operating room; VS: versus; ED: emergency department; SAQ: safety attitudes questionnaire; ANOVA: one-way analysis of variance; HSD: honest significant difference; SD: standard deviation; IQR: interquartile range; SPSS: statistical package for social sciences.

\section{Authors' contributions}

Study conception/design: IB, MK, TS; data collection/analysis: IB, AM; drafting and writing manuscript; IB, MK, AM, TS; acceptation of the final text: IB, MK, AM, TS. All authors read and approved the final manuscript. 


\section{Author details}

${ }^{1}$ School of Health Sciences, University of Tampere, Tampere, Finland. ${ }^{2}$ Pirkanmaa Hospital District, Tampere, Finland. ${ }^{3}$ Faculty of Health Sciences, Klaipeda University, Klaipeda, Lithuania.

\section{Acknowledgements}

This study was partly funded by the University of Tampere, the Finnish Cultural Foundation, and by the Competitive State Research Financing of the Expert Responsibility area of Tampere University Hospital, Grant number 9R048; Grant number 95065.

\section{Competing interests}

The authors declare that they have no competing interests.

Received: 15 February 2016 Accepted: 4 March 2016

Published online: 18 March 2016

\section{References}

1. Thomas EJ, Sexton JB, Neilands TB, Frankel A, Helmreich RL. The effect of executive walk rounds on nurse safety climate attitudes: a randomized trial of clinical units. BMC Health Serv Res. 2005;5:28.

2. Nieva VF, Sorra J. Safety culture assessment: a tool for improving patient safety in healthcare organizations. Qual Saf Health Care. 2003;12(Suppl 2:ii):17-23.

3. Sexton JB, Helmreich RL, Neilands TB, Rowan K, Vella K, Boyden J, Roberts PR, Thomas EJ. The Safety Attitudes Questionnaire: psychometric Properties, Benchmarking Data, and Emerging Research. BMC Health Serv Res. 2006:6(1):44.

4. Singer SJ, Gaba DM, Falwell A, Lin S, Hayes J, Baker L. Patient safety climate in 92 US hospitals: differences by work area and discipline. Med Care. 2009:47(1):23-31.

5. Kaya S, Barsbay S, Karabulut E. The Turkish version of the safety attitudes questionnaire: psychometric properties and baseline data. Qual Saf Health Care. 2010;19(6):572-7.

6. Devriendt E, Van den Heede K, Coussement J, Dejaeger E, Surmont K, Heylen D, Schwendimann R, Sexton B, Wellens NI, Boonen S, Milisen K. Content validity and internal consistency of the Dutch translation of the safety attitudes questionnaire: an observational study. Int J Nurs Stud. 2012:49(3):327-37.

7. De Freitas GF, Hoga LA, Fernandes Mde F, González JS, Ruiz MC, Bonini BB. Brazilian registered nurses perceptions and attitudes towards adverse events in nursing care: a phenomenological study. J Nurs Manag. 2011;19:331-8.

8. The Research Priority Setting Working Group of WHO patient safety: summary of the evidence on patient safety: implications for research. Geneva: WHO. 2008
9. Turunen $H$, Partanen P, Kvist T, Miettinen M, Vehviläinen-Julkunen K. Patient safety culture in acute care: a web-based survey of nurse managers' and registered nurses' views in four Finnish hospitals. Int J Nurs Pract. 2013;19:609-17.

10. Brasaite I, Kaunonen M, Suominen T. Healthcare professionals' knowledge, attitudes and skills regarding patient safety: a systematic literature review. Scand J Caring Sci. 2015;29:30-50.

11. Poley MJ, van der Starre C, van den Bos A, van Dijk M, Tibboel D. Patient safety culture in a Dutch pediatric surgical intensive care unit: an evaluation using the safety attitudes questionnaire. Pediatr Crit Care Med 2011:12:e310-6.

12. Modak I, Sexton JB, Lux TR, Heimreich RL, Thomas EJ. Measuring safety culture in the ambulatory setting: the safety attitudes questionnaireambulatory version. JGIM. 2007;22:1-5.

13. Carney BT, Mills PD, Bagian JP, Weeks WB. Sex differences in operating room care giver perceptions of patient safety: a pilot study from the Veterans Health Administration Medical Team Training Program. Qual Saf Health Care (QSHC). 2010;19(2):128-31.

14. Huang DT, Clermont G, Sexton JB, et al. Perceptions of safety culture vary across the intensive care units of a single institution. Crit Care Med. 2007;35:165e76.

15. Nabhan A, Ahmed-Tawfik MS. Understanding and attitudes towards patient safety concepts in obstetrics. Int J Gynecol Obstet. 2007;98:212-6.

16. Li A-T. Teamwork climate and patient safety attitudes associations among nurses and comparison with physicians in Taiwan. J Nurse Care Qual. 2013;28:60-7.

17. Zimmermann N, Küng K, Sereika SM, Engberg S, Sexton B, Schwendimann R. Assessing the safety attitudes questionnaire (SAQ), German language version in Swiss university hospitals - a validation study. BMC Health Serv Res. 2013;13:347

18. Burns N, Grove SK. The practice of nursing research: appraisal, synthesis, and generation of evidence. St. Louis: Saunders Elsevier; 2009.

19. Helsinki Declaration 2013. WMA Declaration of Helsinki-ethical principles for medical research involving human subjects. Published October 2013. http://www.wma.net/en/30publications/10policies/b3. Accessed 15 Jan 2015.

20. Robson J, de Wet C, McKay J, Bowie P. Do we know what foundation year doctors think about patient safety incident reporting? Development of a web based tool to assess attitude and knowledge. Postgrad Med J. 2012;87:750-6.

21. Uddin SG, Marsteller JA, Sexton JB, Will SE, Fox HE. Provider attitudes toward clinical protocols in obstetrics. Am J Med Qual. 2012;27:335-40.

22. Nilsson L, Lindberget O, Gupta A, Vegfors M. Implementing a pre-operative checklist to increase patient safety: a 1-year follow-up of personnel attitudes. Acta Anaesthesiol Scand. 2010;54:176-82.

\section{Submit your next manuscript to BioMed Central and we will help you at every step:}

- We accept pre-submission inquiries

- Our selector tool helps you to find the most relevant journal

- We provide round the clock customer support

- Convenient online submission

- Thorough peer review

- Inclusion in PubMed and all major indexing services

- Maximum visibility for your research

Submit your manuscript at www.biomedcentral.com/submit
() Biomed Central 